\title{
Effectiveness of dietary interventions using the Mediterranean diet, or any combination of its component food groups in older adults: a systematic of review
}

\author{
J. Lara, L. Errington, M. White, P. J. Moynihan, A. A. Adamson and J. C. Mathers \\ Human Nutrition Research Centre, Institute for Ageing and Health, Newcastle University. Biomedical Research Building, \\ Campus for Ageing and Vitality. Newcastle Upon Tyne, NE4 5PL, UK
}

\begin{abstract}
The health benefits of the Mediterranean diet (MD) have been documented in a growing number of epidemiological studies summarised in previous systematic reviews and meta-analysis ${ }^{(1)}$. However, the effectiveness of intervention studies, as well as the characteristics of effective interventions, promoting the adoption of the MD or any of its food group components has not been documented and the aim of the present study was to address these issues. This study is part of LiveWell, a research programme to develop lifestyle-based interventions to improve healthy ageing at the peri-retirement period. We undertook a systematic review of randomised controlled trials that prospectively evaluated the adoption of a MD or any of its food-group components on healthy and/or "at risk" middle age and aged adult subjects. The protocol for the review has been registered with PROSPERO International prospective register of systematic reviews (CRD42011001484). A search of ten different databases produced 7480 papers that were screened for title and abstract. After abstract and full paper screening, forty-two papers reporting on 18 studies met our criteria of inclusion ie RCT studies reporting a mean or median age between 55 and 70 years, reporting changes in food consumption (or in objective biomarkers of foods consumed, eg $n-3$ fatty acids) after at least 3 months follow up, and with a country of origin classified as high human development by the United Nations Development Programme.

Only three intervention studies on the effectiveness of adopting the MD were identified. These studies employed a low-fat or participants' usual diet as a control group with a follow up ranging from 4 to 12 months. These studies originated from the Netherlands, Spain and Italy. In addition 15 studies reported on interventions promoting consumption of fruits and vegetables or healthy eating compared with control groups commonly receiving minimal- or no-interventions. Two thirds of these studies were carried out in the USA while the rest originated from Japan, Canada, Norway, and UK. Sixteen of these studies reported significant positive effects of the intervention while 2 reported no significant effects. All 18 studies relied on food frequency questionnaires to assess food intake. Heterogeneity of type of the interventions, and length of follow-up was documented. Sensitivity analysis is currently undergoing. This systematic review documents a positive effect of dietary intervention on the MD or its food group components, but also reveals a lack of intervention studies on MD in older adults.

The LiveWell project is funded by the Lifelong Health and Wellbeing Cross-Council Programme initiative in partnership with the UK Health Department: The LLHW Funding Partners are: Biotechnology and Biological Sciences Research Council, Engineering and Physical Sciences Research Council, Economic and Social Research Council, Medical Research Council, Chief Scientist Office of the Scottish Government Health Directorates, National Institute for Health Research /The Department of Health, The Health and Social Care Research \& Development of the Public Health Agency (Northern Ireland), and Wales Office of Research and Development for Health and Social Care, Welsh Assembly Government.
\end{abstract}

1. Sofi F, Abbate R, Gensini GF \& Casini A (2010) Accruing evidence on benefits of adherence to the Mediterranean diet on health: an updated systematic review and meta-analysis. Am J Clin Nutr 92, 1189-96. 